\title{
The additive effect of adherence to multiple healthy lifestyles on subclinical atherosclerosis: Insights from the AWHS
}

\author{
Irina Uzhova, MSc*, Rocio Mateo-Gallego, PhD, Belén Moreno-Franco, PhD, \\ Esther Molina-Montes, PhD, Montserrat Leon-Latre, MD, PhD, \\ José A. Casasnovas Lenguas, PhD, Fernando Civeira, PhD, José L. Peñalvo, PhD
}

Fundación Centro Nacional de Investigaciones Cardiovasculares Carlos III (CNIC), Madrid, Spain (Dr Uzhova); Unidad Clínica y de Investigación en Lípidos y Arteriosclerosis y Laboratorio de Investigación Molecular, Hospital Universitario Miguel Servet, Instituto de Investigación Sanitaria Aragón (IIS Aragón), Zaragoza, Spain (Drs Mateo-Gallego and Civeira); CIBER-CV (Drs Mateo-Gallego and Civeira); Unidad de Prevención Cardiovascular, Instituto Aragonés de Ciencias de la Salud (IACS), Zaragoza, Spain (Drs Moreno-Franco, Leon-Latre, and Casasnovas Lenguas); Departamento de Medicina, Psiquiatría y Dermatología, Universidad de Zaragoza, Zaragoza, Spain (Drs Moreno-Franco and Casasnovas Lenguas); Genetic and Molecular Epidemiology Group, Spanish National Cancer Research Center (CNIO), Madrid, Spain (Dr Molina-Montes); and Friedman School of Nutrition Science and Policy, Tufts University, Boston, MA (Dr Peñalvo)

\section{KEYWORDS:}

Healthy lifestyle;

Atherosclerosis;

Coronary artery calcium;

Atherosclerotic plaque
BACKGROUND: Public health strategies targeting multiple healthy behaviors, rather than individual factors, have been proposed as more efficient strategies to promote cardiovascular health. However, the additive effect of multiple targets on primary prevention has not been fully characterized.

OBJECTIVE: To examine how adherence to multiple healthy behaviors is associated with the presence of subclinical atherosclerosis, a measure of early cardiovascular disease.

METHODS: Analysis of a baseline data from 1798 middle-aged men from the Aragon Workers Health Study conducted between 2009 and 2010. Healthy behaviors were defined according to American Heart Association recommendations, aligned with Spanish Nutritional recommendations and included moderate alcohol consumption, smoking abstinence, no abdominal adiposity, decreased sedentarism, and adherence to Alternate Mediterranean Dietary Index. Presence of coronary artery calcium and plaques in femoral and carotid was quantified by a 16-slice computed tomography scanner and 2D ultrasound.

RESULTS: Moderate alcohol consumption, as well as adherence to Mediterranean diet is independently associated with a $6 \%$ lower risk of having subclinical atherosclerosis. Smoking abstinence is associated with a $11 \%$ lower risk of subclinical atherosclerosis. Those who follow 3 lifestyle behaviors (Mediterranean diet, nonsmoking, and moderate alcohol intake) have $18 \%$ lower odds of
Conflict of interest: All authors read the article, approved the final version, and declared no potential conflict of interest.

Financial Disclosure: The study is funded by FIS (Fondo de Investigaciones Sanitarias) of ISCIII (Instituto de Salud Carlos III), project PI11/ 00403.
* Corresponding author. Fundación Centro Nacional de Investigaciones Cardiovasculares Carlos III (CNIC), Calle de Melchor Fernández Almagro,

3, Madrid, Spain 28029.

E-mail address: irina.uzhova@cnic.es

Submitted September 18, 2017. Accepted for publication March 16, 2018. 
presenting subclinical atherosclerosis compared with those who do not follow these protective lifestyle habits.

CONCLUSION: Adoption of multiple healthy lifestyle behaviors early in life could be a key strategy to tackle the onset of atherosclerosis and reduce cardiovascular disease burden.

(C) 2018 National Lipid Association. All rights reserved.

\section{Introduction}

Atherosclerosis is directly related to coronary heart disease (CHD) and affects a relatively young population. ${ }^{1}$ American Heart Association considered CHD preventable through the lifestyle modifications such as limitation in alcohol consumption, smoking cessation, and physical activity, adherence to healthy diet, healthy weight maintenance, reduced stress, and control of blood cholesterol, blood pressure, and glucose levels. ${ }^{2}$

Studies have shown that unhealthy behaviors are prevalent, with most adults exhibiting more than 1 at a time in what is called a cluster of unhealthy lifestyles. ${ }^{3}$ The US National Health Survey of 16,818 adults revealed that a combination of at least 2 of the following lifestyle behaviors-excessive alcohol, cigarette smoking, overweight or physical inactivity-was prevalent among half of the US population. ${ }^{4}$ Thus, by addressing not only individual behavioral risk factors, but rather their combined effect, intervention strategies for cardiovascular health promotion could enhance their effectiveness.

Few studies have investigated the combined effect of adherence to multiple lifestyle factors on cardiovascular disease (CVD) mortality. Thus, Odegaard et al reported that those having at least 5 protective lifestyle factors (dietary pattern, physical activity, alcohol intake, usual sleep, smoking status, and body mass index) had one-fourth of the risk of CVD mortality compared to those with no protective lifestyle factors. ${ }^{5}$ In another study by Knoops et al, the authors estimated that lack of adherence to healthy lifestyle consisted of Mediterranean dietary pattern, moderate alcohol consumption, physical activity, and being nonsmoker accounted for a $64 \%$ of deaths from CHD and $61 \%$ from CVD. ${ }^{6}$ In terms of assessing how those lifestyle factors are associate with the early stage of the CHD most previous studies investigated the relationship between the combination of lifestyle behaviors and coronary artery calcification $(\mathrm{CAC}){ }^{7-9}$ or intimamedia thickness (IMT) ${ }^{10,11}$ as markers of subclinical atherosclerosis. ${ }^{12} \mathrm{CAC}$ score (CACS) is considered a major predictor of $\mathrm{CHD},{ }^{13}$ a score of 0 being associated with only a $1 \%$ probability of having a cardiac event, ${ }^{14}$ whereas an elevated score, above 0 , has been shown to be associated with a 10fold increase in the risk of a cardiac event. ${ }^{13}$ Noteworthy, the limitation of CACS as a marker of atherosclerosis is that it can only be identified at late stages of disease progression, which makes it inadequate for its use in asymptomatic individuals. With respect to IMT, some studies have suggested a weak correlation with coronary atherosclerosis. ${ }^{15}$ To date, there are a limited number of studies exploring the association between direct indicators of atherosclerosis, such as the presence of atherosclerotic plaques, and adherence to lifestyle behaviors. Moreover, most of them focus on atherosclerotic plaques located in carotid arteries. Assessment of femoral plaque compared with carotid might potentially become a useful tool in predicting CHD as it has been shown to be better correlated with traditional CVD risk factors. ${ }^{16}$ Therefore, in this study we aim to explore the relationship between adherence to multiple healthy lifestyle behaviors and presence of plaques in the femoral artery as well as other markers of subclinical atherosclerosis.

\section{Methods}

\section{Study participants}

The Aragon Workers Health Study (AWHS) is a longitudinal cohort study aiming to investigate the determinants of the development and progression of metabolic abnormalities and subclinical atherosclerosis among CVD-free middle-aged population. The participants were recruited between February 2009 and December 2010. The details of the study are described in detail elsewhere. ${ }^{17}$ The present study was performed on a sample of 1933 participants aged 40-55 y with complete baseline dietary, lifestyle and imaging data. Owing to the low percentage of females in AWHS and recognizing the differential effect of gender on health behaviors, we excluded female participants $(\mathrm{N}=135,0.5 \%$ of the sample). The final sample available for analysis was of 1798. The Central Institutional Review Board of Aragón approved the study, and written informed consent was obtained from all study participants.

\section{Assessment of lifestyle behaviors}

Information on lifestyle behaviors were collected using food frequency questionnaire and physical activity questionnaires previously validated for Spain. ${ }^{18,19}$ Dietary energy, macronutrient and micronutrient intakes were derived using the Spanish food composition tables. ${ }^{2,21}$ The variables used in the analyses were defined as follows: (1) alcohol: taking into account that there is no universal definition for moderate alcohol consumption, we have examined the evidence from several studies, ${ }^{22-24}$ dietary guidelines for Spanish population, which includes optional moderate alcohol intake in the form of wine or beer, ${ }^{25}$ recommendations given by American Heart Association, ${ }^{26}$ and considered the following categories for alcohol 
consumption: nondrinkers or excess drinkers (more than 30 grams of ethanol/d) vs moderate drinkers (no more than 30 grams and not less than 10 grams of ethanol/d); (2) smoking: nonsmokers combined with ex-smokers vs current smokers; (3) physical activity: as a proxy for a lack of physical activity we used the sitting time (h/d). Two categories were used-spending less vs more than 5.5 hours a day sitting. Cutoff was based on the First tertile of sitting time as well as previous work by the previously published work by Dr Leon-Latre within AWHS cohort, where it was observed that those who on average spend $5.5 \mathrm{~h} / \mathrm{d}$ and less sitting had lower glucose, and c-reactive protein, whereas those who is being sedentary for $5.5 \mathrm{~h} /$ $\mathrm{d}$ and more had increased body mass index (BMI), waist circumference (WC), and insulin level ${ }^{27}$; (4) diet: we used the Alternate Mediterranean Dietary Index (aMED) score. ${ }^{28}$ Two categories were used: aMED score from 0 to 4 vs aMED score from 5 to 9 (cutoff based on the median of the sample). Healthy lifestyle behaviors were defined as follows ${ }^{26}$ : moderate alcohol consumption, nonsmoking, normal WC $(\leq 102 \mathrm{~cm}),{ }^{29}$ sitting time less than $5.5 \mathrm{~h} / \mathrm{d}$, and aMED score higher than 5 . The variables for combined lifestyle analysis were considered as follows: (1) aMED score more than 5 and moderate alcohol consumption vs aMED score less than 5 and nondrinkers/ excess drinkers (category used as a reference); (2) aMED score more than 5, moderate alcohol consumption, and nonsmoking vs aMED score less than 5, nondrinkers or excess drinkers, and current smoking (category used as a reference); (3) aMED score more than 5, moderate alcohol consumption, nonsmoking, and no abdominal adiposity vs aMED score less than 5, nondrinkers or excess drinkers, current smoking, and abdominal adiposity (category used as a reference); (4) aMED score more than 5, moderate alcohol consumption, nonsmoking, no abdominal adiposity, and sitting time less than $5.5 \mathrm{~h} / \mathrm{d}$ vs aMED score less than 5 , nondrinkers or excess drinkers, current smoking, abdominal adiposity, and sitting time more than $5.5 \mathrm{~h} / \mathrm{d}$ (category used as a reference).

\section{Assessment of subclinical atherosclerosis}

A 16-slice computed tomography scanner (Philips Brillians, Philips Healthcare, Andover, MA) with noncontract prospective electrocardiography-gated acquisition and Agatston equation was used to quantify CACS. ${ }^{30}$ The following categories of CACS were computed: CACS $=0$ and CACS $>0 .{ }^{31}$ The assessment of atherosclerotic plaques was performed in bilateral carotid and femoral arteries using 2D ultrasound (Philips iU22 ultrasound, Philips Healthcare, Bothell, Washington) by a cross-sectional sweep of aforementioned territories. Plaque was defined as a "focal structure that protrudes into the lumen of the carotid artery at least $0.5 \mathrm{~mm}$ or $\geq 50 \%$ thicker than the surrounding IMT". ${ }^{32}$ Presence of subclinical atherosclerosis was defined as the presence of at least 1 plaque in any of the territories and/or CACS $>0$.

\section{Clinical, anthropometric, and laboratory data and other variables}

Protocols for data collection are described in detail elsewhere. ${ }^{17}$ The data on medication use, hospitalization occurred over a past year, anthropometric measurements, blood pressure, and heart rate were collected at the clinical examination. Samples of fasting blood and urine were collected and stored for laboratory analysis. Data on sociodemographic and lifestyle variables were self-reported and included age, level of complete education, working shifts, smoking status, and sitting time. Traditional CVD risk factors were defined according to European guidelines as follows: blood pressure $\geq 140 / 90 \mathrm{~mm} \mathrm{Hg}$ (130/80 in participants with diabetes) or current use of antihypertensive medication determined the presence of hypertension ${ }^{33}$; total cholesterol $\geq 190 \mathrm{mg} / \mathrm{dL}$ $(4.9 \mathrm{mmol} / \mathrm{L})$ or use of medication determined a presence of hypercholesterolemia ${ }^{34}$; a fasting serum glucose $\geq 126 \mathrm{mg} /$ $\mathrm{dL}(7.0 \mathrm{mmol} / \mathrm{L})$, or a $\mathrm{HbA} 1 \mathrm{c} \geq 6.5 \%$ or use of medication determined the presence of diabetes. ${ }^{35}$

\section{Statistical analysis}

T-test for continuous variables and chi-square test for categorical variables were used to compare the data between categories. The major demographic, lifestyle, and dietary factors related to subclinical atherosclerosis and its markers were identified by backward stepwise logistic regression. Each block was run independently and the results of the model were unadjusted. Multivariate adjusted Poisson regression models with robust estimates were used to assess the association between lifestyle behaviors (assessing its individual as well as additive effects) and presence of plaques, CACS, and subclinical atherosclerosis. The inclusion of covariates in the model was based on the assessment of sociodemographic, clinical, and lifestyle variables. Those variables that significantly differed between the groups in the descriptive analysis $(P<.05)$ as well as significantly contributed to the presence of the atherosclerosis and its markers identified by backward stepwise logistic regression model were included in the model. The fully adjusted model included age (years), education (incomplete high school degree/high school degree/college degree), hypertension (yes/no), hypercholesterolemia (yes/no), and diabetes (yes/ no). The number of lifestyle behaviors (cont.) and presence of subclinical atherosclerosis was assessed by fully adjusted Poisson regression models with robust estimates. All statistical analyses were undertaken using SPSS v. 23 (IBM Corp., Armonk, NY).

\section{Results}

Participants presenting at least one plaques in carotid or femoral territories and increased CACS were more likely to be older, have lower educational level, being current smokers, present hypercholesterolemia, diabetes, 
hypertension, as well as increased BMI and WC. Accordingly, these participants tended to have higher levels of total cholesterol, low-density lipoprotein cholesterol, triglycerides, apolipoprotein B, HbA1c, HOMA-IR, glucose and insulin level, as well as lower apolipoprotein A1 and highdensity lipoprotein cholesterol levels (Table 1). Moreover, they were less likely to engage in moderate to vigorous physical activity, more likely to have increased alcohol consumption, and smoke (16-17 cigarettes a day on average). However, those participants presenting plaque in the carotids had a higher adherence to Mediterranean dietary pattern, whereas those with plaques in the femorals, or CACS $>0$ were more likely to score lower on aMED scale.

The main factors related to the presence of subclinical atherosclerosis were mainly increased age, and traditional CVD risk factors, such as hypertension, hypercholesterolemia, and diabetes (Table 2). An age increase was associated with significant increase in the risk of having plaque in carotids, femorals, CACS $>0$, and subclinical atherosclerosis $(P<.001)$. Higher education status was significantly associated with $24 \%(P=.013)$ lower risk of plaque in femorals $(P<.001)$. Among traditional CVD risk factors, smoking, and hypertension were the main factors related to the presence of subclinical atherosclerosis (odds ratio $[\mathrm{OR}]=3.41$, 95\% confidence interval $[\mathrm{CI}]: 2.29 ; 5.06 ; \mathrm{OR}=3.62,95 \%$ CI: $2.32 ; 5.63)$. Hypertension was the main factor related to the presence of plaque in carotids and CACS $>0(\mathrm{OR}=1.94,95 \%$ CI: $1.57 ; 2.41 ; \mathrm{OR}=2.08,95 \%$ CI: $1.68 ; 2.58)$, whereas smoking determined the presence of plaque in femorals ( $\mathrm{OR}=3.13,95 \%$ CI: $2.53 ; 3.88)$. Assessment of the dietary factors showed that presence of plaque in femorals is less likely to be related to increased fruits $(\mathrm{OR}=0.63,95 \% \mathrm{CI}$ : $0.52,0.77)$, whole grains consumption $(\mathrm{OR}=0.72,95 \% \mathrm{CI}$ : 0.60, 0.86), and higher MUFA/SFA ratio (OR $=0.79,95 \%$ CI: $0.65,0.96)$. Excessive alcohol consumption was the main determinant of subclinical atherosclerosis $(\mathrm{OR}=1.63,95 \% \mathrm{CI}: 1.18 ; 2.25)$ and its markers $\left(\mathrm{OR}_{\text {femorals }}=1.47,95 \% \mathrm{CI}: 1.21 ; 1.78\right.$; $\mathrm{OR}_{\text {carotids }}=1.39,95 \% \mathrm{CI}: 1.14 ; 1.70 ; \quad \mathrm{OR} \quad \mathrm{CACS}$ $>_{0}=1.48,95 \%$ CI: $1.22 ; 1.80$ ).

The analysis of the individual lifestyle factors (Table 3) showed that being nonsmoker significantly associated with a lower risk of having atherosclerosis in all territories (prevalence ratio $[\mathrm{PR}]_{\text {femorals }}: 0.64 ; 95 \%$ CI: $0.60 ; 0.69 ; \mathrm{PR}_{\text {carotids: }}$ : $0.81 ; 95 \%$ CI: $0.71 ; 0.91 ; \mathrm{PR}_{\mathrm{CACS}>0}: 0.82 ; 95 \%$ CI: 0.73 ; 0.92; $\mathrm{PR}_{\text {subatherosclerosis }}: 0.89 ; 95 \%$ CI: 0.86; 0.93). Adherence to Mediterranean diet is associated with lower risk of having plaque in femorals (PR: 0.84; 95\% CI: 0.78; $0.92)$ and decreased odds of presenting subclinical atherosclerosis (PR: 0.94; 95\% CI: 0.91; 0.99); while moderate alcohol consumption is associated with lower risk of having atherosclerosis in all territories $\left(\mathrm{PR}_{\text {femorals }}\right.$ : 0.90; 95\% CI: $0.83 ; 0.98 ; \mathrm{PR}_{\mathrm{CACS}}>0$ : 0.84; 95\% CI: 0.74; 0.95; PR $_{\text {subatherosclerosis: }}$ PR: 0.94; 95\% CI: 0.89; 0.98), except for having a plaque in carotid.

The analysis of the association between potential synergic effects of lifestyle factors and disease revealed that the combination of an adherence to Mediterranean dietary pattern, moderate alcohol intake, and being nonsmoker is associated with $47 \%(P<.001), 35 \%(P .01)$, and $18 \%$ $(P<.01)$ lower risk of having femoral plaque, CACS $>0$, and subclinical atherosclerosis. Complying with any 3 out of 5 healthy lifestyle behaviors is associated with $16 \%$ lower prevalence of subclinical atherosclerosis $(\mathrm{PR}=0.84,95 \% \mathrm{CI}=0.73,0.99, P<.05)($ Fig. 1$)$.

\section{Discussion}

In this analysis, we demonstrated that adherence to multiple healthy lifestyles has an additive effect on subclinical atherosclerosis measured by direct quantification of the presence of plaques. Consumption of alcohol in moderation, being nonsmoker, and an adherence to a Mediterranean dietary pattern, was associated with a $47 \%$ lower prevalence of plaques in the femoral territory, $35 \%$ lower risk of CACS $>0$, and overall to an $18 \%$ lower prevalence of subclinical atherosclerosis.

In our study, the moderate alcohol consumption was observed to be associated with lower risk of having plaque in femorals, CACS, and subclinical atherosclerosis. It has been shown that moderate alcohol intake is associated with a decline in fibrinogen, and lipoprotein (a) and increase in apolipoprotein A1 and high-density lipoprotein concentrations, ${ }^{36}$ which together might promote an antiatherogenic effect. Prospective study reported a J-shaped relationship between alcohol intake and CVD mortality and showing that moderate alcohol consumers had lower risk than either abstainers or heavy drinkers. ${ }^{37}$ Our findings also support these results to some extent. Once abstainers were included in the category of moderate drinking behavior we observed that the risk of having CACS $>0$ increased by $4 \%$, whereas the risk of subclinical atherosclerosis increased by $3 \%$ and the results became nonsignificant (Supplemental Table 1). Therefore, we could assume that alcohol abstainers might have a slightly higher risk of subclinical atherosclerosis compared with moderate alcohol consumers. Cultural aspects as well as the type of alcohol typically consumed in the area where the study was conducted might be an explanation for the observed protective effect of alcohol. In Spain, one of the major types of alcohol typically consumed is wine, which is known for its cardiovascular health-promoting benefits. Another aspect is that its consumption traditionally takes place mostly during the meal, which in some studies has been observed to offer more protection against atherosclerotic disease. ${ }^{38}$ Surprisingly, the risk of having a plaque in the carotid arteries significantly decreased by $16 \%$ in the fully adjusted model, once alcohol abstainers were included in the category of moderate drinkers. These findings could be partially explained by the dietary behavior of alcohol abstainers, as they were observed to have the lowest intake of sodium, processed meat, together with the highest intakes of fiber and fruits. Although the cardioprotective effect of higher fiber and 


\begin{tabular}{|c|c|c|c|c|c|c|c|c|}
\hline \multirow[b]{3}{*}{ Baseline characteristics } & \multicolumn{2}{|c|}{ Plaque in femorals } & \multicolumn{2}{|c|}{ Plaque in carotids } & \multicolumn{2}{|l|}{ CACS } & \multicolumn{2}{|c|}{ Plaque and CACS } \\
\hline & No plaque & $\geq 1$ plaque & No plaque & $\geq 1$ plaque & $\mathrm{CACS}=0$ & $\mathrm{CACS}>0$ & $\begin{array}{l}\text { No plaque and } \\
\text { CACS }=0\end{array}$ & $\begin{array}{l}\geq 1 \text { plaque } \\
\text { and } C A C S>0\end{array}$ \\
\hline & $N=834$ & $N=1115$ & $\mathrm{~N}=1238$ & $N=709$ & $N=1190$ & $N=759$ & $N=232$ & $N=556$ \\
\hline \multicolumn{9}{|l|}{ Sociodemographics } \\
\hline \multicolumn{8}{|l|}{ Education level } & $52.6 \pm 3.07^{\star}$ \\
\hline$<$ High school degree & $405(48.6)$ & $617(55.4)$ & $619(50.0)$ & $403(56.8)$ & $592(49.8)$ & $430(56.7)$ & $99(42.7)$ & $315(56.7)$ \\
\hline High school degree & $76(9.10)$ & $130(11.7)$ & $141(11.4)$ & $65(9.20)^{*}$ & $126(10.6)$ & $80(10.5)$ & $28(12.1)$ & $56(10.1)$ \\
\hline College degree & $43(5.20)$ & $34(3.10)^{*}$ & $56(4.50)$ & $21(3.00)^{*}$ & $50(4.20)$ & $27(3.60)$ & $15(6.50)$ & $20(3.60)^{*}$ \\
\hline \multicolumn{9}{|l|}{ Working shift } \\
\hline Day shift & $67(8.00)$ & $62(5.60)$ & $83(6.70)$ & $46(6.50)$ & $72(6.10)$ & $57(7.50)$ & $20(8.60)$ & $41(7.40)$ \\
\hline Rotation shift & $691(82.9)$ & $927(83.1)$ & $1038(83.8)$ & $578(81.5)$ & $1002(84.2)$ & $616(81.2)$ & $191(82.4)$ & $450(80.9)$ \\
\hline Night shift & $76(9.10)$ & $126(11.3)$ & $117(9.50)$ & $85(12.0)$ & $116(9.70)$ & $86(11.3)$ & $21(9.10)$ & $65(11.7)$ \\
\hline \multicolumn{9}{|l|}{ Traditional CVD factors } \\
\hline Hypercholesterolemia, n (\%) & $633(82.3)$ & $909(88.3)^{*}$ & $951(83.5)$ & $590(89.8)^{*}$ & $919(84.4)$ & $623(87.9)^{*}$ & $172(80.0)$ & $458(88.1)^{\star}$ \\
\hline Total cholesterol (mg/dL) & $220 \pm 36.0$ & $223 \pm 35.6^{*}$ & $220 \pm 35.6$ & $225 \pm 36.0^{*}$ & $221 \pm 35.6$ & $223 \pm 36.1$ & $216 \pm 35.0$ & $224 \pm 35.9^{*}$ \\
\hline LDL cholesterol (mg/dL) & $137 \pm 31.5$ & $139 \pm 32.7$ & $137 \pm 31.7$ & $141 \pm 32.9^{*}$ & $138 \pm 31.3$ & $139 \pm 33.6$ & $133 \pm 31.0$ & $140 \pm 33.8^{*}$ \\
\hline HDL cholesterol (mg/dL) & $54.4 \pm 11.5$ & $51.2 \pm 10.8^{*}$ & $53.3 \pm 11.7$ & $51.3 \pm 10.2^{*}$ & $53.2 \pm 11.7$ & $51.6 \pm 10.4^{*}$ & $54.3 \pm 10.9$ & $51.6 \pm 10.2^{*}$ \\
\hline Triglycerides (mg/dL) & $142 \pm 88.3$ & $164 \pm 106^{*}$ & $150 \pm 99.4$ & $164 \pm 99.0^{*}$ & $150 \pm 97.7$ & $162 \pm 102^{*}$ & $142 \pm 93.6$ & $161 \pm 102^{*}$ \\
\hline Apolipoprotein A1 & $147 \pm 19.4$ & $142 \pm 18.2^{*}$ & $145 \pm 19.3$ & $143 \pm 17.9^{*}$ & $145 \pm 19.0$ & $142 \pm 18.5^{*}$ & $146 \pm 17.4$ & $142 \pm 18.4^{*}$ \\
\hline Apolipoprotein B & $103 \pm 22.6$ & $108 \pm 22.0^{*}$ & $104 \pm 22.3$ & $109 \pm 22.3^{*}$ & $104 \pm 22.2$ & $108 \pm 22.4^{*}$ & $99.0 \pm 23.3$ & $110 \pm 22.6^{*}$ \\
\hline $\begin{array}{l}\text { Apolipoprotein A1/ } \\
\text { apolipoprotein B }\end{array}$ & $0.71 \pm 0.19$ & $0.77 \pm 0.19^{*}$ & $0.73 \pm 0.19$ & $0.77 \pm 0.19^{*}$ & $0.73 \pm 0.19$ & $0.77 \pm 0.19^{*}$ & $0.69 \pm 0.19$ & $0.79 \pm 0.19^{*}$ \\
\hline Hypertension, n (\%) & $176(22.9)$ & $333(32.4)^{*}$ & $263(23.1)$ & $246(37.4)^{*}$ & $239(21.9)$ & $270(38.1)^{*}$ & $29(13.5)$ & $177(34.0)^{*}$ \\
\hline $\mathrm{SBP}(\mathrm{mm} \mathrm{Hg})$ & $123 \pm 12.9$ & $128 \pm 14.7^{*}$ & $124 \pm 13.7$ & $128 \pm 14.5^{*}$ & $124 \pm 13.3$ & $128 \pm 15.0^{*}$ & $121 \pm 11.6$ & $127 \pm 14.5^{*}$ \\
\hline $\mathrm{DBP}(\mathrm{mm} \mathrm{Hg})$ & $81.9 \pm 9.09$ & $83.9 \pm 9.52^{*}$ & $82.4 \pm 9.37$ & $84.2 \pm 9.33^{*}$ & $82.5 \pm 9.24$ & $83.9 \pm 9.55^{*}$ & $80.7 \pm 8.45$ & $83.7 \pm 9.20^{*}$ \\
\hline Diabetes, n (\%) & $27(3.50)$ & $77(7.50)^{*}$ & $49(4.30)$ & $55(8.40)^{*}$ & $38(3.50)$ & $66(9.30)^{*}$ & $7(3.30)$ & $41(7.90)^{*}$ \\
\hline Glucose (mg/dL) & $97.9 \pm 16.1$ & $98.8 \pm 18.4$ & $97.5 \pm 16.0$ & $100 \pm 19.6^{*}$ & $97.4 \pm 15.6$ & $100 \pm 19.9^{*}$ & $98.6 \pm 20.7$ & $98.9 \pm 17.6$ \\
\hline Insulin $(\mu \mathrm{U} / \mathrm{mL})$ & $7.38 \pm 5.44$ & $8.25 \pm 5.96^{*}$ & $7.60 \pm 5.68$ & $8.35 \pm 5.88^{*}$ & $7.66 \pm 5.54$ & $8.20 \pm 6.06$ & $7.20 \pm 5.37$ & $7.74 \pm 5.75$ \\
\hline HOMA_IR & $1.86 \pm 1.59$ & $2.09 \pm 1.82^{*}$ & $1.90 \pm 1.69$ & $2.14 \pm 1.78^{*}$ & $1.90 \pm 1.59$ & $2.13 \pm 1.91^{*}$ & $1.84 \pm 1.65$ & $1.99 \pm 1.76$ \\
\hline HOMA_B & $81.8 \pm 69.9$ & $93.5 \pm 80.0^{*}$ & $86.4 \pm 74.5$ & $92.1 \pm 78.5$ & $87.4 \pm 70.4$ & $90.0 \pm 83.7$ & $76.2 \pm 48.4$ & $83.3 \pm 65.4$ \\
\hline $\mathrm{HbA1c}$ & $5.49 \pm 0.49$ & $5.61 \pm 0.60^{*}$ & $5.52 \pm 0.52$ & $5.61 \pm 0.62^{*}$ & $5.52 \pm 0.53$ & $5.61 \pm 0.59^{*}$ & $5.45 \pm 0.59$ & $5.59 \pm 0.57^{*}$ \\
\hline Abdominal obesity, n (\%) & $227(28.3)$ & $356(32.8)^{*}$ & $341(28.7)$ & $242(34.8)^{*}$ & $329(28.7)$ & $254(34.3)^{*}$ & $54(24.3)$ & $158(29.2)$ \\
\hline Waist circumference $(\mathrm{cm})$ & $96.9 \pm 9.12$ & $98.3 \pm 8.82^{*}$ & $97.1 \pm 8.84$ & $98.7 \pm 9.12^{*}$ & $97.1 \pm 8.90$ & $98.6 \pm 9.00^{*}$ & $95.5 \pm 9.07$ & $97.6 \pm 8.61^{*}$ \\
\hline Body mass index (BMI) $\left(\mathrm{kg} / \mathrm{m}^{2}\right)$ & $27.6 \pm 3.41$ & $27.9 \pm 3.33$ & $27.6 \pm 3.25$ & $28.1 \pm 3.55^{*}$ & $27.6 \pm 3.34$ & $28.1 \pm 3.38^{*}$ & $27.1 \pm 3.11$ & $27.8 \pm 3.26^{*}$ \\
\hline $30>\mathrm{BMI} \geq 25$ & $464(56.0)$ & $640(57.5)$ & $700(56.8)$ & $404(57.1)$ & $657(55.4)$ & $447(59.0)^{*}$ & $128(55.4)$ & $341(61.4)^{*}$ \\
\hline $\mathrm{BMI} \geq 30$ & $186(22.4)$ & $264(23.7)$ & $273(22.2)$ & $176(24.9)$ & $263(22.2)$ & $187(24.7)^{*}$ & $43(18.6)$ & $118(21.3)^{*}$ \\
\hline \multicolumn{9}{|l|}{ Lifestyle } \\
\hline MPA (h/wk) & $3.76 \pm 5.23$ & $2.84 \pm 4.67^{*}$ & $3.45 \pm 5.12$ & $2.86 \pm 4.58^{*}$ & $3.35 \pm 4.96$ & $3.06 \pm 4.90$ & $3.86 \pm 5.28$ & $3.03 \pm 4.85^{*}$ \\
\hline VPA (h/wk) & $1.11 \pm 2.89$ & $0.84 \pm 2.55^{*}$ & $1.05 \pm 2.84$ & $0.79 \pm 2.44^{*}$ & $1.00 \pm 2.75$ & $0.90 \pm 2.63$ & $1.36 \pm 3.10$ & $0.93 \pm 2.68$ * \\
\hline
\end{tabular}


fruit intakes and salt restriction is well known, processed meat consumption on a regular basis was recently shown to be associated with the slightly higher risk of developing heart disease. ${ }^{39}$ Not only healthier dietary behavior, but lower plasma concentration of triglycerides together with decreased systolic and diastolic blood pressure observed among this group of participants might have resulted in a beneficial effect of alcohol restriction on the presence of an atherosclerotic plaque in carotid arteries.

Adherence to a Mediterranean dietary pattern in our study was significantly associated with a lower prevalence of plaques in the femoral arteries and subclinical atherosclerosis, but not with CACS, which was rather unexpected taking into account the fact that there is extensive evidence demonstrating the beneficial role of a Mediterranean diet in prevention of CVD. ${ }^{40}$ We hypothesized that a lack of an association with CACS be due to the homogeneity of the dietary habits of our sample, as it was observed in our previous research. ${ }^{41}$ Interestingly, participants with at least 1 plaque in the carotid arteries were observed to have better adherence to a Mediterranean dietary pattern as well as higher total cholesterol concentration, low-density lipoprotein cholesterol, increased presence of abdominal obesity, excessive alcohol consumption, and being current smokers. Thus, we could hypothesize that these participants have changed their diet during the past year to address the health-related issues, and therefore, we see the lack of the association with respect to diet, or those factors mentioned previously have overcome a cardioprotective effect of an adherence to a Mediterranean dietary pattern.

Participants presenting at least 3 of 5 cardioprotective behaviors including moderate alcohol consumption, smoking abstinence, and adherence to a Mediterranean diet had an $18 \%$ lower odds of presenting subclinical atherosclerosis. Our findings are in line with MESA study, which reported a $46 \%$ lower risk of having increased CAC in those who never smoked, maintained a healthy body weight, performed $>150 \mathrm{~min} / \mathrm{wk}$ of moderate or $>75 \mathrm{~min} /$ wk of vigorous physical activity, and followed a Mediterranean dietary pattern. ${ }^{42}$ Another prospective cohort showed that an overall healthy behavior including moderate alcohol consumption along with smoking abstinence, presence of $\mathrm{WC}<95 \mathrm{~cm}$, daily physical activity (walking/bicycling $\geq 40 \mathrm{~min} / \mathrm{d}$ and exercising $\geq 1 \mathrm{~h} / \mathrm{wk}$ ), and presenting higher Recommended Food Score is responsible for the prevention of $79 \%$ of the myocardial infarction events. ${ }^{43}$ A point to consider is that the adherence to this healthy lifestyle pattern in our study was not prevalent accounting for only $5.4 \%$ of the population presenting "ideal healthy lifestyle". Supposedly this might reflect either the lack of successful public health interventions targeting middle-age men or the existence of barriers to adopt this ideal lifestyle. Taking into account the amount of time spent at the workplace, these findings are essential for workplace health promotion professionals and might serve as an important key message for public health interventions. 
Table 2 Demographic, lifestyle, and dietary factors related to the presence of plaques, CACS $>0$, and subclinical atherosclerosis

\begin{tabular}{|c|c|c|c|c|c|c|}
\hline \multirow[b]{2}{*}{ Factor } & \multirow[b]{2}{*}{ Categories } & \multirow[b]{2}{*}{$\mathrm{N}(\%)$} & Plaque in femorals & Plaque in carotid & CACS $>0$ & Subclincial atherosclerosis \\
\hline & & & \multicolumn{4}{|l|}{ OR $95 \%$ CI } \\
\hline \multicolumn{7}{|l|}{ Demographic factors } \\
\hline \multirow{4}{*}{ Age } & $40-44 y$ & $124(6.9)$ & (ref) & (ref) & (ref) & (ref) \\
\hline & $45-49 y$ & $442(24.6)$ & $1.83(1.20 ; 2.79)$ & $2.96(1.14 ; 3.38)$ & $2.30(1.33 ; 4.13)$ & $3.18(1.49 ; 6.80)$ \\
\hline & $50-54 y$ & $814(45.3)$ & $3.11(2.11 ; 4.73)$ & $4.14(2.46 ; 6.95)$ & $5.30(3.08 ; 9.12)$ & $8.71(4.15 ; 18.5)$ \\
\hline & $\geq 55 \mathrm{y}$ & $418(23.2)$ & $3.73(2.43 ; 5.71)$ & $5.07(2.97 ; 8.66)$ & $7.61(4.35 ; 13.3)$ & $11.6(5.29 ; 15.6)$ \\
\hline \multirow[t]{3}{*}{ Education } & $<$ High school degree & $937(52.1)$ & (ref) & & & (ref) \\
\hline & High school degree & $190(10.6)$ & $1.18(0.85 ; 1.63)$ & & & \\
\hline & College degree & $670(37.3)$ & $0.73(0.60 ; 0.94)$ & & & $0.66(0.46 ; 0.94)$ \\
\hline \multicolumn{7}{|l|}{ Traditional CVD factors } \\
\hline \multirow[t]{2}{*}{ Smoking } & No & & (ref) & (ref) & (ref) & (ref) \\
\hline & Yes & $1153(64.1)$ & $3.13(2.53 ; 3.88)$ & $1.38(1.13 ; 1.70)$ & $1.33(1.09 ; 1.63)$ & $3.41(2.29 ; 5.06)$ \\
\hline \multirow[t]{2}{*}{ Hypertension } & No & & (ref) & (ref) & (ref) & (ref) \\
\hline & Yes & $1289(71.7)$ & $1.66(1.33 ; 2.08)$ & $1.94(1.57 ; 2.41)$ & $2.08(1.68 ; 2.58)$ & $3.62(2.32 ; 5.63)$ \\
\hline \multirow[t]{2}{*}{ Hypercholesterolemia } & No & & (ref) & (ref) & (ref) & (ref) \\
\hline & Yes & $1542(85.8)$ & $1.68(1.27 ; 2.22)$ & $1.71(1.27 ; 2.31)$ & $1.30(0.98 ; 1.73)$ & $1.82(1.15 ; 2.85)$ \\
\hline \multirow[t]{2}{*}{ Diabetes } & No & & (ref) & (ref) & (ref) & \\
\hline & Yes & $104(5.80)$ & $1.81(1.13 ; 2.89)$ & $1.61(1.07 ; 2.43)$ & $2.25(1.47 ; 3.42)$ & \\
\hline \multicolumn{7}{|l|}{ aMED score components } \\
\hline \multirow{2}{*}{ Fruits } & Less than a median & & (ref) & & & \\
\hline & More than a median & $886(49.3)$ & $0.63(0.52 ; 0.77)$ & & & \\
\hline \multirow[t]{2}{*}{ Whole grains } & Less than a median & & (ref) & & & \\
\hline & More than a median & $792(44.0)$ & $0.72(0.60 ; 0.86)$ & & & \\
\hline \multirow[t]{2}{*}{ Fish } & Less than a median & & & & & \\
\hline & More than a median & $863(48.0)$ & & & & \\
\hline \multirow[t]{2}{*}{ MUFA/SFA } & Less than a median & & (ref) & & & \\
\hline & More than a median & $885(49.2)$ & $0.79(0.65 ; 0.96)$ & & & \\
\hline \multirow[t]{2}{*}{ Red meat } & Less than a median & & & & & \\
\hline & More than a median & $887(49.3)$ & & & & \\
\hline \multirow[t]{2}{*}{ Alcohol } & Less than a median & & (ref) & (ref) & (ref) & (ref) \\
\hline & More than a median & $739(41.1)$ & $1.47(1.21 ; 1.78)$ & $1.39(1.14 ; 1.70)$ & $1.48(1.22 ; 1.80)$ & $1.63(1.18 ; 2.25)$ \\
\hline
\end{tabular}

CACS, coronary artery calcification score; MUFA, monounsaturated fatty acids; SFA, saturated fatty acids; OR, odds ratio; CI, confidence interval; ref, reference.

Each model (each block) was run independently. Unadjusted models.

Determinants identified on the basis of a backward stepwise logistic regression model. All variables are categorical. 
Table 3 Adjusted prevalence ratios PR $(95 \% \mathrm{CI})^{\dagger}$ for the presence of plaques, CACS $>0$, and subclinical atherosclerosis by individual and combined lifestyle behaviors and adiposity

\begin{tabular}{|c|c|c|c|c|}
\hline Lifestyle behaviors and adiposity & Plaque femoral & Plaque carotid & CACS $>0$ & Atherosclerosis \\
\hline \multicolumn{5}{|l|}{ Individual behaviors } \\
\hline$\uparrow$ Diet & $0.84(0.78 ; 0.92) *$ & $1.03(0.91 ; 1.16)$ & $0.92(0.82 ; 1.03)$ & $0.94(0.91 ; 0.99)^{* *}$ \\
\hline$\downarrow$ Alcohol & $0.90(0.83 ; 0.98)^{* * *}$ & $1.04(0.92 ; 1.18)$ & $0.84(0.74 ; 0.95)^{* *}$ & $0.94(0.89 ; 0.98) * *$ \\
\hline$\downarrow$ Smoking & $0.64(0.6 ; 0.69)^{*}$ & $0.81(0.71 ; 0.91)^{*}$ & $0.82(0.73 ; 0.92)^{*}$ & $0.89(0.86 ; 0.93)^{*}$ \\
\hline$\downarrow$ Adiposity & $0.97(0.89 ; 1.06)$ & $0.93(0.82 ; 1.06)$ & $1.00(0.88 ; 1.12)$ & $1.02(0.97 ; 1.06)$ \\
\hline$\downarrow$ Sedentarism & $0.93(0.86 ; 1.01)$ & $0.92(0.82 ; 1.04)$ & $1.02(0.91 ; 1.14)$ & $0.97(0.93 ; 1.02)$ \\
\hline \multicolumn{5}{|l|}{ Multiple behaviors } \\
\hline$\uparrow$ Diet & $0.84(0.78 ; 0.92)^{*}$ & $1.03(0.91 ; 1.16)$ & $0.92(0.82 ; 1.03)$ & $0.93(0.89 ; 0.98)^{* *}$ \\
\hline$\uparrow$ Diet $+\downarrow$ Alcohol & $0.77(0.68 ; 0.87)^{*}$ & $1.04(0.87 ; 1.23)$ & $0.79(0.66 ; 0.93)^{* *}$ & $0.89(0.84 ; 0.95)^{* *}$ \\
\hline$\uparrow$ Diet $+\downarrow$ Alcohol $+\downarrow$ Smoking & $0.53(0.46 ; 0.62)^{*}$ & $0.95(0.76 ; 1.18)$ & $0.65(0.52 ; 0.81)^{*}$ & $0.82(0.75 ; 0.88)^{*}$ \\
\hline $\begin{array}{l}\uparrow \text { Diet }+\downarrow \text { Alcohol }+\downarrow \text { Smoking } \\
+\downarrow \text { Adiposity }\end{array}$ & $0.51(0.42 ; 0.62)^{*}$ & $0.94(0.68 ; 1.29)$ & $0.63(0.46 ; 0.87)^{* *}$ & $0.83(0.75 ; 0.92)^{* *}$ \\
\hline $\begin{array}{l}\uparrow \text { Diet }+\downarrow \text { Alcohol }+\downarrow \text { Smoking } \\
+\downarrow \text { Adiposity }+\downarrow \text { Sedentarism }\end{array}$ & $0.43(0.33 ; 0.58)^{*}$ & $0.86(0.57 ; 1.29)$ & $0.69(0.45 ; 1.04)$ & $0.82(0.71 ; 0.95)^{* *}$ \\
\hline
\end{tabular}

Our study has several worth mentioning strengths and limitations. One of the major strength of our study is the possibility to evaluate the effect of a healthy lifestyle on atherosclerosis in middle-aged asymptomatic individuals, who would be the ideal candidates for primary prevention as they have not developed the advance stage of the disease yet. It is worth mentioning the choice of the statistical model in our analysis. Recently the criticism has been

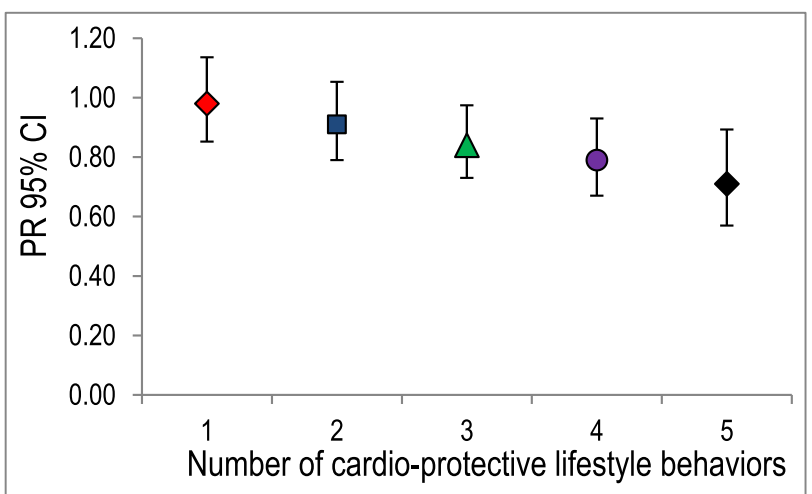

Figure 1 Prevalence ratio $(95 \% \mathrm{CI})$ for the presence of subclincial atherosclerosis according to the number of cardioprotective lifestyle behaviors. Description of the variable "number if cardioprotective lifestyle behaviors": (1) 1 of 5 protective lifestyle behaviors are present: either having low WC, or moderate alcohol consumption, or nonsmoking, or sitting time less than $5.5 \mathrm{~h} / \mathrm{d}$ or having aMED score 5 or more; (2) random combination of 2 of 5 protective behaviors are present etc.; * Poisson regression model with robust estimate is adjusted for age, education, diabetes, hypertension, and hypercholesterolemia. PR, prevalence ratio; CI, confidence interval. voiced regarding the appropriate assessment of risk in cross-sectional studies showing that the estimates obtained by logistic regression in studies with a highly prevalent disease outcome would greatly overestimate the association. ${ }^{44}$ Therefore, we have chosen to use Poisson regression to estimate the prevalence ratio of adherence to lifestyle behaviors with disease outcome. A major limitation of our study is its cross-sectional design and inability to eliminate the presence of confounding factors, which might explain the reason we see the different degree of the effect in the relationship among lifestyle habits and atherosclerosis in different territories as well as the lack of the contribution of sedentarism and adiposity to the disease risk. Even though multivariable adjustment was applied, still due to the nature of the study the effect of residual confounding factors could not be ruled out. The use of food frequency questionnaire as the assessment method of dietary intake, which relied on the participants memory to recall typical food intake over the past year is another limitation, which might have introduced the error in dietary data. The elimination of female participants from the study limited the generalizability of our results to only male population. Further studies should replicate our analysis including both sexes. Finally, the restricted number of participants with "ideal healthy lifestyle" is another limitation, which could have affected the power of the true association.

From a public health perspective, our findings are of particular importance for health professionals in the clinical settings working with primary prevention and aiming to tackle CVD at its earliest development. Formulating a simple message highlighting the importance to avoid 
smoking, follow healthy eating pattern, and consume alcohol in moderation could be an easy solution to prevent coronary artery disease at an early stage.

\section{Conclusion}

Consumption of alcohol in moderation, being nonsmoker, and an adherence to a Mediterranean dietary pattern, accounts for the $18 \%$ lower prevalence of subclinical atherosclerosis. Highlighting the importance of a healthy lifestyle promotion and introducing this message by health professionals could be a key strategy to tackle the atherosclerosis burden at its earliest development.

\section{Acknowledgments}

We would like to thank all study participants and the personnel at the General Motors factory for the collaboration. José L. Peñalvo is currently an employee at Merck KGaA.

Authors' contributions: I.U. contributed to conception and design, analysis and interpretation, and drafted the article; R.M.G. contributed to acquisition and interpretation, and critically revised the article; B.M.F. contributed to acquisition and interpretation, and critically revised the article; E.M.M. contributed to interpretation, and critically revised the article; M.L.L. contributed to acquisition and interpretation, and critically revised the article; J.A.C.L. contributed to acquisition, and critically revised the article; J.L.P. contributed to conception and design, acquisition and interpretation, and critically revised the article.

\section{References}

1. Mozaffarian D, Benjamin EJ, Go AS, et al. Heart disease and stroke statistics-2015 update: a report from the American Heart Association. Circulation. 2015;131:e29-e322.

2. Kromhout D, Menotti A, Kesteloot H, Sans S. Prevention of coronary heart disease by diet and lifestyle. Circulation. 2002;105: 893-898.

3. Fine LJ, Philogene GS, Gramling R, Coups EJ, Sinha S. Prevalence of multiple chronic disease risk factors: 2001 National Health Interview Survey. Am J Prev Med. 2004;27:18-24.

4. Coups EJ, Gaba A, Orleans CT. Physician screening for multiple behavioral health risk factors. Am J Prev Med. 2004;27:34-41.

5. Odegaard AO, Parpia B, Chen J. Combined lifestyle factors and cardiovascular disease mortality in Chinese mean and women. Circulation. 2011;124(25):2847-2854.

6. Knoops KT. Mediterranean diet, lifestyle factors, and 10-year mortality in elderly European men and women: the HALE project. JAMA. 2004;292(12):1433-1439.

7. Mamudu HM, Paul TK, Wang L, et al. The effects of multiple coronary artery disease risk factors on subclinical atherosclerosis in a rural population in the United States. Prev Med. 2016;88: 140-146.

8. Cheong E, Lee JY, Lee SH, et al. Lifestyle including dietary habits and changes in coronary artery calcium score: a retrospective cohort study. Clin Hypertens. 2015;22:5.
9. Lee C-D, Jae SY. Lifestyle Factors and Coronary Artery Calcification. Pulse. 2015;2:95-102.

10. Santos IS, Goulart AC, Pereira AC, Lotufo PA, Benseñor IM. Association between Cardiovascular Health Score and Carotid Intima-Media Thickness: Cross-Sectional Analysis of the Brazilian Longitudinal Study of Adult Health (ELSA-Brasil) Baseline Assessment. J Am Soc Echocardiogr. 2017;29:1207-1216.e4.

11. Jhamnani S, Patel D, Heimlich L, et al. Meta-Analysis of the Effects of Lifestyle Modifications on Coronary and Carotid Atherosclerotic Burden. Am J Cardiol. 2015;115:268-275.

12. Spring B, Moller AC, Colangelo LA, et al. Healthy lifestyle change and subclinical atherosclerosis in young adults: Coronary Artery Risk Development in Young Adults (CARDIA) study. Circulation. 2014;130:10-17.

13. Detrano R, Guerci A, Carr J, Bild D. Coronary calcium as a predictor of coronary events in four racial or ethnic groups. Engl J. 2008; 358(13):1336-1345.

14. Sarwar A, Shaw L, Shapiro M, Blankstein R. Diagnostic and prognostic value of absence of coronary artery calcification. JACC Cardiovasc. 2009;2(6):675-688.

15. Simon A, Chironi G. The relationship between carotid intima-media thickness and coronary atherosclerosis revisited. Eur Heart J. 2007; 28:2049-2050.

16. Laclaustra M, Casasnovas JA, Fernández-Ortiz A, et al. Femoral and Carotid Subclinical Atherosclerosis Association With Risk Factors and Coronary Calcium: The AWHS Study. J Am Coll Cardiol. 2016; 67:1263-1274.

17. Casasnovas JA, Alcaide V, Civeira F, et al. Aragon workers' health studydesign and cohort description. BMC Cardiovasc Disord. 2012;12:45.

18. Martin-moreno JM, Boyle P, Gorgojo L, et al. Development and validation of a food frequency questionnaire in Spain. Int $J$ Epidemiol. 1993;22:512-519.

19. Martínez-González MA, López-Fontana C, Varo JJ, Sánchez-Villegas A, Martinez JA. Validation of the Spanish version of the physical activity questionnaire used in the Nurses' Health Study and the Health Professionals' Follow-up Study. Public Health Nutr. 2005;8:920-927.

20. Moreiras O, Carvajal A, Cabrera L, Cuadrado C. Tablas de composición de alimentos, 2011. Madrid: Pirámide; 2011.

21. Mataix J. Tabla de composición de alimentos (Food composition tables). Granada: Granada Univ.; 2003.

22. Baliunas DO, Taylor BJ, Irving H, et al. Alcohol as a risk factor for type 2 diabetes. Diabetes Care. 2009;32:2123-2132.

23. Corrao G, Rubbiati L, Bagnardi V, Zambon A, Poikolainen K. Alcohol and coronary heart disease: a meta-analysis. Addiction. 2000;95: $1505-1523$.

24. Ronksley PE, Brien SE, Turner BJ, Mukamal KJ, Ghali WA. Association of alcohol consumption with selected cardiovascular disease outcomes: a systematic review and meta-analysis. BMJ. 2011;342:d671.

25. Aranceta Bartrina J, Arija Val V, Maíz Aldalur E, et al. Grupo Colaborativo dela Sociedad Española de Nutrición Comunitaria (SENC). Guías alimentarias para la población española (SENC, diciembre 2016); la nueva pirámide de la alimentación saludable. Nutr Hosp. 2016;33(S8):1-48.

26. Lichtenstein AH, Appel LJ, Brands M, et al. Summary of American Heart Association diet and lifestyle recommendations revision 2006. Arterioscler Thromb Vasc Biol. 2006;26:2186-2191.

27. León-Latre M, Moreno-Franco B, Andrés-Esteban EM, et al. Sedentary lifestyle and its relation to cardiovascular risk factors, insulin resistance and inflammatory profile. Rev Esp Cardiol (Engl Ed). 2014;67(6):449-455.

28. Fung TT, Rexrode KM, Mantzoros CS, et al. Mediterranean diet and incidence of and mortality from coronary heart disease and stroke in women. Circulation. 2009;119:1093-1100.

29. Williams L. Third report of the National Cholesterol Education Program (NCEP) expert panel on detection, evaluation, and treatment of high blood cholesterol in adults (Adult Treatment Panel III) final report. Circulation. 2002;106:3143-3421. 
30. Agatston AS, Janowitz WR, Hildner FJ, et al. Quantification of coronary artery calcium using ultrafast computed tomography. J Am Coll Cardiol. 1990;15:827-832.

31. Martin SS, Blaha MJ, Blankstein R, et al. Dyslipidemia, coronary artery calcium, and incident atherosclerotic cardiovascular disease: Implications for statin therapy from the multi-ethnic study of atherosclerosis. Circulation. 2014;129:77-86.

32. Muntendam P, McCall C, Sanz J, et al. The BioImage Study: novel approaches to risk assessment in the primary prevention of atherosclerotic cardiovascular disease—study design and objectives. Am Heart J. 2010;160:49-57.

33. Perk J, De Backer G, Gohlke H, et al. European Guidelines on cardiovascular disease prevention in clinical practice (version 2012) The Fifth Joint Task Force of the European Society of Cardiology and Other Societies on Cardiovascular Disease Prevention in Clinical Practice (constituted by representatives of nine societies and by invited experts). Eur Heart J. 2012;33: 1635-1701.

34. Reiner Ž, Catapano AL, De Backer G, et al. ESC/EAS Guidelines for the management of dyslipidaemias The Task Force for the management of dyslipidaemias of the European Society of Cardiology (ESC) and the European Atherosclerosis Society (EAS). Eur Heart J. 2011;32:1769-1818.

35. Association AD. Diagnosis and classification of diabetes mellitus. Diabetes Care. 2010;33:S62-S69.

36. Brien SE, Ronksley PE, Turner BJ, Mukamal KJ, Ghali WA. Effect of alcohol consumption on biological markers associated with risk of coronary heart disease: systematic review and meta-analysis of interventional studies. BMJ. 2011;342:d636.

37. Di Castelnuovo A, Costanzo S, Bagnardi V, Donati MB, Iacoviello L, de Gaetano G. Alcohol dosing and total mortality in men and women. Arch Intern Med. 2006;166(22):2437-2445.

38. Criqui MH, Ringel BL. Does diet or alcohol explain the French paradox? Lancet. 1994;344:1719-1723.

39. Micha R, Wallace SK, Mozaffarian D. Red and processed meat consumption and risk of incident coronary heart disease, stroke, and diabetes mellitus. A systematic review and meta-analysis. Circulation. 2010;121(21):2271-2283.

40. Martinez-Gonzalez MA, Bes-Rastrollo M. Dietary patterns, Mediterranean diet, and cardiovascular disease. Curr Opin Lipidol. 2014;25:20-26.

41. Peñalvo JL, Oliva B, Sotos-Prieto M, et al. Greater adherence to a Mediterranean dietary pattern is associated with improved plasma lipid profile: the Aragon Health Workers Study cohort. Rev Esp Cardiol (Engl Ed). 2015;68:290-297.

42. Ahmed HM, Blaha MJ, Nasir K, et al. Low-risk lifestyle, coronary calcium, cardiovascular events, and mortality: results from MESA. Am J Epidemiol. 2013;178:12-21.

43. Åkesson A, Larsson SC, Discacciati A, Wolk A. Low-risk diet and lifestyle habits in the primary prevention of myocardial infarction in men: a population-based prospective cohort study. J Am Coll Cardiol. 2014;64:1299-1306.

44. Barros AJD, Hirakata VN. Alternatives for logistic regression in crosssectional studies: an empirical comparison of models that directly estimate the prevalence ratio. BMC Med Res Methodol. 2003;3:21. 


\section{Appendix}

Supplemental Table 1 Adjusted prevalence ratio $(95 \% \mathrm{CI})^{\dagger}$ for the presence of plaques in femorals and carotids, CACs $>0$ and presence of noncoronary and subclinical atherosclerosis by alcohol consumption (nondrinkers combined with moderate alcohol consumers)

\begin{tabular}{|c|c|c|c|c|c|}
\hline Individual behaviors & Plaque femoral & Plaque carotid & $\begin{array}{l}\text { Noncoronary } \\
\text { atherosclerosis }\end{array}$ & $\mathrm{CAC}>0$ & Atherosclerosis \\
\hline$\downarrow$ Alcohol & $0.90(0.83 ; 0.98)^{* *}$ & $0.87(0.77 ; 0.99)^{* *}$ & $0.97(0.93 ; 1.02)$ & $0.88(0.78 ; 0.98)^{* *}$ & $0.97(0.93 ; 1.01)$ \\
\hline
\end{tabular}

